\title{
Biocompatible Synthetic Tracheal Matrices Based on Polymer Ultra-Fibrous Materials Colonized by MesenchymäMultipotent Cells
}

D01: $10.17691 / \mathrm{stm} 2016.8 .1 .01$

Received August 25, 2015

M.V. Kiselevskiy, MD, DSc, Professor, Head of Cell Immunity Laboratory';

N.Y. Anisimova, MD, DSc, Leading Researcher, Cell Immunity Laboratory';

E.A. Kornushenkov, PhD, Head of the Department of Experimental Therapy';

A.D. Shepelev, PhD, Engineer-Researcher, Laboratory of Biocompatible Matrices;

S.N. Chvalun, DSc, Professor, Head of the Department of Polymer Materials;

B.E. Polotskiy, MD, DSc, Professor, Leading Researcher, Thoracoabdominal Department";

M.I. Davydov, MD, DSc, Professor, Academician of the Russian Academy of Sciences, Director ${ }^{1}$

${ }^{1}$ N.N. Blokhin Russian Cancer Research Center, 24 Kashirskoe Shosse, Moscow, 115478, Russian Federation; ${ }^{2}$ National Research Center "Kurchatov Institute", 1 Academic Kurchatov Square, Moscow, 123182,

Russian Federation

The aim of the investigation is to study biocompatibility and biodegradation of synthetic tracheal matrices based on ultra-fibrous polymer materials, colonized by mesenchymal multipotent stromal cells (MMSC) of recipient.

Materials and Methods. The study was performed on beagle dogs, which underwent heterotopic implantation of synthetic tracheal scaffolds made of a nonwoven material by electrospinning and colonized by the recipient MMSC. Implant biocompatibility was assessed by computed tomography and macro- and microstructure analysis of the extracted implant and adjacent tissues.

Results. It was established that the developed trachea matrix specimens implanted heterotopically to the dogs preserved biocompatibility, ensured colonization by recipient cells, did not cause local and systemic reactions of rejection, and had no general toxic effect. According to the data obtained there was made a conclusion on the biocompatibility of tracheal matrices made of the polymer ultra-fibrous materials and their prospective applications as bioimplants for trachea defect replacements.

Key words: tracheal matrix; heterotopic implantation; biocompatibility; mesenchymal multipotent cells.

Various defects of trachea due to malignant neoplasms, traumas and congenital abnormalities require sometimes its resection. Meanwhile, formation of primary "end-to-end" anastamosis is feasible less than in $50 \%$ patients with this pathology [1-3]. In other cases application of implants is required to replace significant tracheal defects. At present there are no prostheses in clinical practice, matching the native trachea by their biological, configurative and mechanical properties. The most perspective direction in creating trachea bioimplants is a tissue engineering approach [1-6]. The first successful transplantation of the bioengineering construction, made of decellularized donor trachea colonized by recipient cells, was performed to the patient with a terminal stage of bronchomalatia in 2008 [7]. As an application of donor trachea has a number of limitations, and decellularization procedure may impair mechanical properties of implants and is rather labor-consuming, various synthetic matrices based on nanocomposite or ultra-fibrous materials capable to be populated by mesenchimal multipotent stromal cells (MMSC) of the bone marrow have recently appeared for this purpose [3, 8-10]. Despite the evident progress, the attempts to create a construction combining optimal mechanical properties, chemical stability and structure, providing colonization by recipient cells and

For contacts: Mikhail V. Kiselevskiy, e-mail: kisele@inbox.ru 
intercellular interactions have not been a success so far. Therefore further investigations directed to the creation of hybrid synthetic and natural matrices, combining optimal mechanical properties and stability with biocompatibility.

The aim of the investigation is to assess biocompatibility, biodegradation and ability to colonize synthetic trachea matrices made of ultra-fibrous polymer materials by recipient cells.

Materials and Methods. Synthetic tracheal matrices for investigations on dogs were obtained from nonwoven material using proprietory pilot single capillary electrospinning system. Fluoroplastic F-42V (copolymer of tetrafluoroethylene with vinylidene fluoride) (ChimCombinat, Russia) was used as a polymer for the non-woven material. Rings, reinforcing the non-woven polymeric material, were produced by thermopressing, and polyurethane Elastillan $1195 \mathrm{~A}$ (Elastogran, Germany) was used as a basic material (Figure 1).

Mechanical properties of the specimens were tested using a universal testing machine Instron 5965 (Instron, USA) provided with a computer system for on-line data analysis.

The studies were conducted on 6 beagle dogs ( 1 female and 5 males) aging $4.0 \pm 1.2$ years, housed in vivarium and Department of Experimental Therapy of N.N. Blokhin Russian Cancer Research Center. All manipulations with the animals were performed in accordance with the ethical principles established by European Convention for the Protection of Vertebrata used for Experimental and other Scientific Purposes (the Convention was passed in Strasburg, March, 18, 1986, adopted in Strasburg, June, 15, 2006) and the requirements of the Declaration of Helsinki (the Declaration was passed in Helsinki, Finland, June, 1964, and revised in October, 2000, Edinburg, Scotland), and the World Medical Association (2000).

To improve biocompatibility and to boost matrix integration, tracheas were colonized with MMSC of dogs-recipients. MMSC were generated from the bone marrow cells, obtained by sternal puncture. The marrow cells were placed into sterile culture tubes with nutrition medium RPMI 1640 (PanEco, Russia), containing 10\% of embryonal fetal serum (PanEco, Russia). Two passages were performed to remove nonadhesive cells.

The analysis of MMSC culture was performed with the help of inverted microscope Axiovert 40 (Carl Zeiss, Germany) in a transmitted-light or phase contrast mode. Histologic examination was done in compliance with the standard technique, sections were stained with hematoxylin and eosin (PanEco, Russia), preparations were examined and photographed using light microscope Axioplan 2 (Carl Zeiss, Germany). The level of superficial MMSC marker expression was determined by flow cytometry by means of cytofluorometer BD FACS Canto II (Becton Dickinson, USA) using antibodies

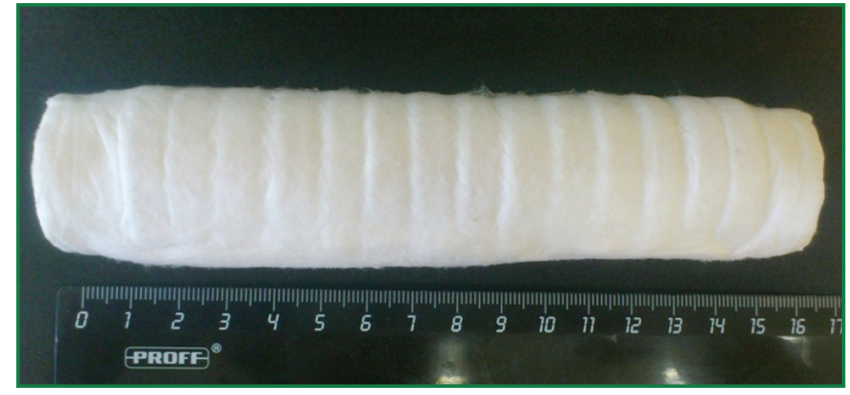

Figure 1. Tracheal matrix specimen based on polymer ultrafibrous materials for the experiments on dogs

CD45, CD90, CD105 conjugated with fluorochromes (Becton Dickinson, USA).

Sterile matrices were populated by incubation with the suspension of MMSC in the cultural medium at $37^{\circ} \mathrm{C}$ and $4.5 \% \mathrm{CO}_{2}$ for 7 days, shaking periodically the cultural tube. Colonization of the matrix by MMSC was controlled by obtaining imprints from the surface of the implant fragments on the slide, covered by polylysine (Thermo Scientific, USA) with the following Romanovsky-Giemsa staining [11].

Heterotopic implantation of tracheal matrices, populated by autologous MMSC, was performed ex vivo on 6 beagle dogs: matrices were implanted in the intermuscular space of the thigh fold. For this purpose an intermuscular pocket, into which a matrix sample was placed, was formed in the dogs, then the wound edges were closed in layers by interrupted sutures, the external suture and adjacent skin area were treated with antiseptics. The operations were performed under general anesthesia observing all the requirements of aseptics and antiseptics in the operating unit of the Department of Experimental Therapy of N.N. Blokhin Russian Cancer Research Center.

Animals have been observed for a month. During this period a daily visual examination of the operative area was conducted, regular analysis of hematologic indices was performed using an analyzer (ProCyte Dx, The Netherlands), which has been especially calibrated to investigate the dog's blood. Dogs-recipients underwent computed tomography on SOMATOM Sensation 64 System (Siemens, Germany). Various planes of the lumbosacral region were analyzed in layers covering the area of xiphoid process. 30-35 days after the implantation specimens of the matrix were taken out under general anesthesia for microscopic and histologic examination.

To estimate the degree of significance of the revealed changes the analysis of a set of related individualized samples using Friedman test and "Nonparametric statistics" module of the Statistica 6.0 (StatSoft) program was performed. Differences were considered significant at $p<0.05$

Results and Discussion. The specimens of the 
tracheal synthetic matrices had a tubular shape and did not essentially differ from the native tracheas of the dogs by their dimensions and mechanical properties. The differences did not exceed $30 \%$ (Tables 1 and 2 ).

Before the sterile matrices were implanted to the animals they were colonized ex vivo by MMSC, obtained from the bone marrow cells of the recipients by cultivation in the nutrition media. Target cells were presented mainly by large polygonal or spindle cells with long processes

Table 1

Mean values of dimensional characteristics of the native dog trachea and matrices $(\mathrm{mm})$

\begin{tabular}{|ccc|}
\hline Parameters of the semiring & Native trachea & Matrix \\
\hline Width & $4.0 \pm 0.3$ & $4.0 \pm 0.2$ \\
\hline Length & $64.0 \pm 2.3$ & $63.0 \pm 1.1$ \\
\hline Thickness & $2.0 \pm 0.3$ & $2.0 \pm 0.1$ \\
\hline Diameter & $23.0 \pm 9.0$ & $26.0 \pm 5.0$ \\
\hline
\end{tabular}

being in contact with each other. The cell nuclei were moderately oxyphilic of the round or oval form with centrally located nucleoli. The whole area of the tube bottom was covered with a relatively uniform confluent cellular monolayer (Figure 2). Immunophenotype analysis of the $2^{\text {nd }}$ passage cells established, that the subpopulation of $\mathrm{CD} 45^{-} \mathrm{CD} 90^{+} \mathrm{CD} 105^{+}$cells amounted to not less than $65 \%$.

The morphological examinations and the assessment of the immunophenotype of the cells allowed us to characterize them as MMSC.

The analysis of the imprints from the matrix surfaces made it possible to characterize the revealed MMSC: they were distinguished by the enlarged nuclei and had colony-forming foci, indicating to the preservation of the proliferative potential of MMSC in the tracheal matrices (Figure 3).

Tracheal matrices populated by MMSC were implanted heterotopically to the dogs in the intermuscular pocket formed in the inguinal area (Figure 4).

Complete blood count was made periodically to

Table 2

Elasticity of matrix fragments, reinforced by polyurethane-based semirings, and the native trachea

\begin{tabular}{|c|c|c|c|c|}
\hline \multirow{2}{*}{ Parameters of statistical analysis } & \multicolumn{2}{|c|}{$\begin{array}{l}\text { Multiple compression - index } \\
\text { of the developing load fall (\%) }\end{array}$} & \multicolumn{2}{|c|}{$\begin{array}{l}\text { Single compression - index } \\
\text { of the ring rigidity }(\mathrm{kPa})\end{array}$} \\
\hline & Matrix & Native trachea & Matrix & Native trachea \\
\hline Median & 8.2 & 7.0 & 43.4 & 37.2 \\
\hline Minimum & 7.6 & 6.3 & 38.2 & 32.2 \\
\hline Maximum & 8.6 & 8.2 & 47.2 & 41.0 \\
\hline First quartile ( $25^{\text {th }}$ percentile) & 7.8 & 6.3 & 39.2 & 32.2 \\
\hline Third quartile ( $75^{\text {th }}$ percentile) & 8.6 & 8.2 & 44.0 & 41.0 \\
\hline Comparison with the native trachea min-max $(\%)$ & \multicolumn{2}{|c|}{$6-20$} & \multicolumn{2}{|c|}{$4-28$} \\
\hline
\end{tabular}
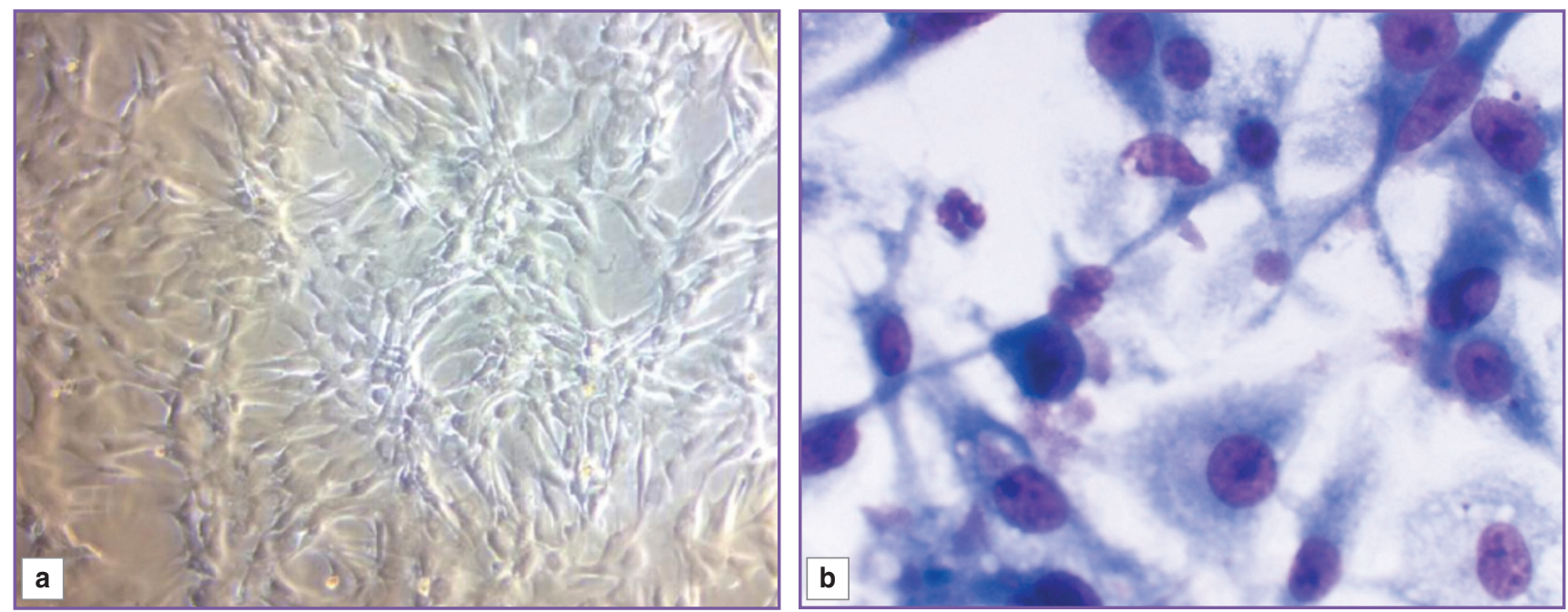

Figure 2. Morphology of dogs' mesenchymal multipotent stromal cells in the culture: (a) live culture, $\times 200$; (b) RomanovskyGiemsa cell staining, $\times 900$ 

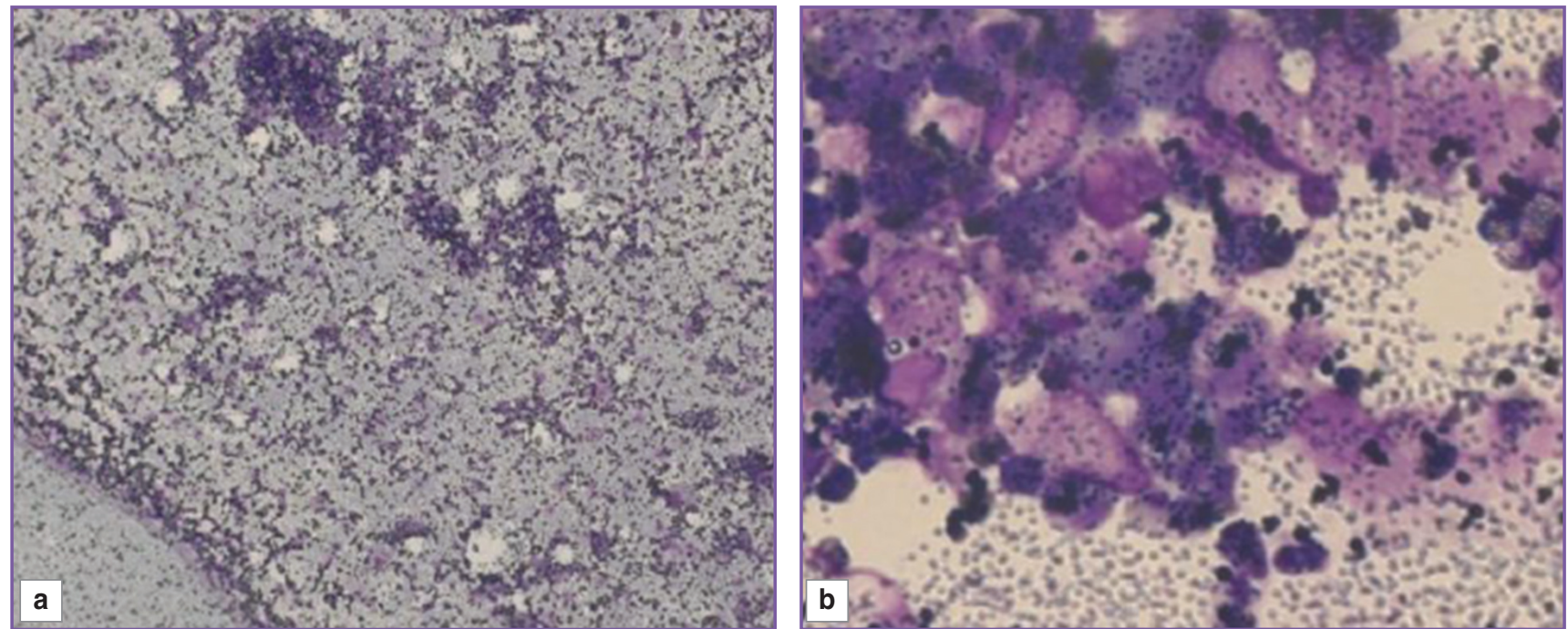

Figure 3. Cell composition of the matrix surface: (a) $\times 200$; (b) $\times 900$. Romanovsky-Giemsa cell staining
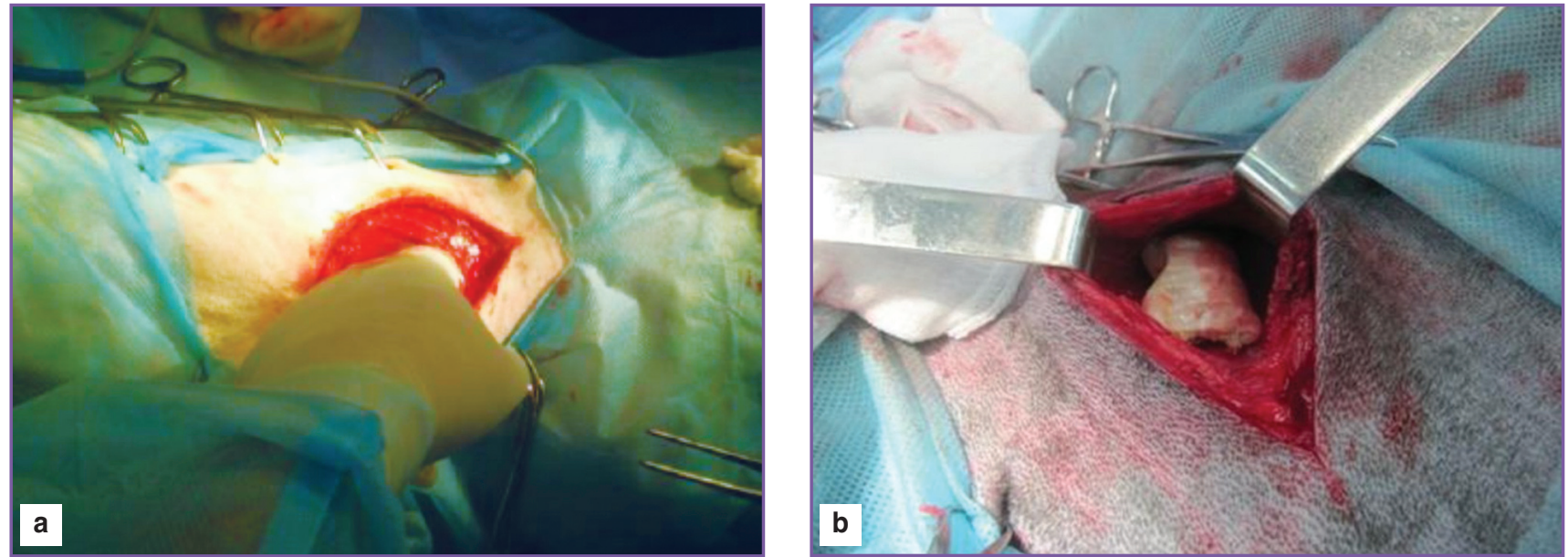

Figure 4. The main stages of heterotopic transplantation of matrix specimens loaded with mesenchymal multipotent stromal cells to the dogs: (a) intermuscular pocket formation; (b) implanted tracheal matrix specimen

monitor systemic reaction of implant rejection or inflammation following heterotopic implantation of the experimental specimens. Leukogram (WBC) and absolute leucocyte count were estimated, as changes in these hematological indices directly correlate with the development of the cell reaction cascade, implementing transplantat rejection $[12,13]$. Hematological indices of animals were shown to vary within the physiological norm during a month after the implantation (Table 3), i.e. no leukocytosis showing the signs of systemic reaction of rejection were observed.

20-25 days after the operation the animals underwent computed tomography under general anesthesia. The data obtained by computed tomography led to the conclusion that experimental specimens in the animal organisms did not lose such signs of functionality as preservation of matrix framework providing the inner lumen of almost a circular shape, and physical integrity (Figure 5).
Visual examination did not reveal any local reaction of rejection: edema, suppuration, reddening, fistulas, massive growth of fibrous tissue in the implantation area during the whole period of observation (Figure $6(\mathrm{a})$ ).

The tracheal matrix, removed from the inguinal fold a month after the implantation, preserved its shape and framework properties, and was covered with connective tissue capsule, intimately bound to the matrix tissue (Figure 6 (b)). Morphological investigations of the adjacent tissues revealed no inflammatory exudates in the implantation area. No signs of inflammatory leukocyte infiltration, which forms a cellular cascade of rejection in case of acute rejection, realizing cell destruction of the allograft, were found either [13].

A powerful colonization by the recipient cells with formation of vessels and connective tissue fibers extending from the surface deep into the implant was found in the extracted tracheal matrix alongside with 
Table 3

Statistical analysis of the dogs' hematological index dynamics after heterotopic transplantation of tracheal matrix specimens, loaded with autologous mesenchymal multipotent stromal cells

\begin{tabular}{|c|c|c|c|c|c|}
\hline \multirow{2}{*}{ Blood indices } & \multirow{2}{*}{$\begin{array}{l}\text { Statistical } \\
\text { values }\end{array}$} & \multirow{2}{*}{$\begin{array}{c}\text { Before } \\
\text { the operation }\end{array}$} & \multicolumn{2}{|c|}{ After the operation } & \multirow{2}{*}{$\begin{array}{l}\text { p (Friedman } \\
\text { test) }\end{array}$} \\
\hline & & & After 2 weeks & After 4 weeks & \\
\hline \multirow{3}{*}{ Erythrocytes $\left(\times 10^{12} / \mathrm{L}\right)$} & Median & 6.2 & 6.6 & 7.0 & \multirow[t]{3}{*}{0.441} \\
\hline & Min & 5.7 & 6.0 & 5.9 & \\
\hline & Max & 8.1 & 8.0 & 8.1 & \\
\hline \multirow{3}{*}{ Hemoglobin $(\mathrm{g} / \mathrm{dl})$} & Median & 14.7 & 14.6 & 15.9 & \multirow[t]{3}{*}{0.052} \\
\hline & Min & 13.1 & 11.7 & 13.8 & \\
\hline & Max & 16.4 & 16.0 & 17.7 & \\
\hline \multirow{3}{*}{ Leukocytes (×10\%/L) } & Median & 9.5 & 10.2 & 11.2 & \multirow[t]{3}{*}{0.097} \\
\hline & Min & 8.5 & 9.8 & 9.4 & \\
\hline & Max & 9.9 & 15.4 & 14.5 & \\
\hline \multirow{3}{*}{ Neutrophils (\%) } & Median & 69.3 & 76.7 & 72.0 & \multirow[t]{3}{*}{0.716} \\
\hline & Min & 66.3 & 64.2 & 64.8 & \\
\hline & Max & 71.5 & 79.8 & 77.3 & \\
\hline \multirow{3}{*}{ Lymphocytes (\%) } & Median & 27.6 & 21.5 & 24.6 & \multirow[t]{3}{*}{0.717} \\
\hline & Min & 26.3 & 16.1 & 21.6 & \\
\hline & $\operatorname{Max}$ & 31.5 & 34.6 & 32.1 & \\
\hline \multirow{3}{*}{ Monocytes (\%) } & Median & 0.2 & 0.2 & 0.1 & \multirow[t]{3}{*}{0.368} \\
\hline & Min & 0.1 & 0.1 & 0.1 & \\
\hline & Max & 0.2 & 0.2 & 0.2 & \\
\hline \multirow{3}{*}{ Eosinophils (\%) } & Median & 2.0 & 1.5 & 3.0 & \multirow[t]{3}{*}{0.497} \\
\hline & Min & 2.0 & 1.0 & 1.0 & \\
\hline & Max & 4.0 & 4.0 & 3.0 & \\
\hline \multirow{3}{*}{ Basophils (\%) } & Median & 0 & 0.1 & 0.1 & \multirow[t]{3}{*}{0.135} \\
\hline & Min & 0 & 0 & 0 & \\
\hline & Max & 0.1 & 0.2 & 0.3 & \\
\hline
\end{tabular}

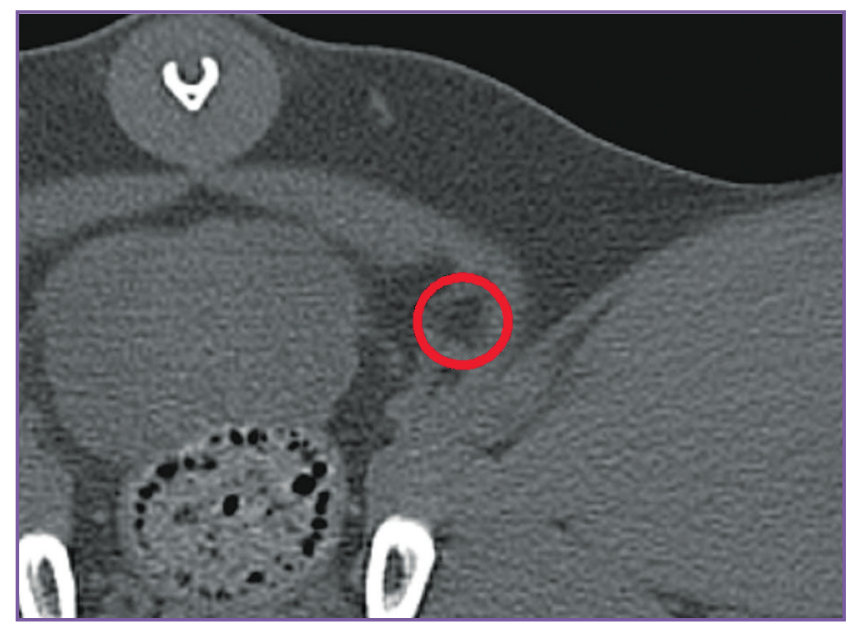

Figure 5. Computed tomography image of the tracheal matrix made of ultra-fibrous fluoroplastic on day 25 after heterotopic transplantation to the dog (craniocaudal projection) the preservation of the ultra-fibrous structure (Figure 7). The data gained by CT, macro- and micromorphological examination showed that the shape, framework and constituent elements (undestroyed fibers of fluoroplastic and intact polyurythane rings) of the synthetic matrices were preserved and there were no signs of biodegradation of the tested experimental specimens.

Conclusion. Examination of the synthetic material specimens, colonized by mesenchimal multipotent stromal cells demonstrated preservation of physical properties (size, design, integrity), functional characteristics (framework, strength, elasticity), initial structure (regular fibers of the matrix base, reinforces by semirings), and absence of local and systemic signs of rejection within a month when implanted heterotopically. The specimens also preserved the integrity and lumen potency, provided their colonization by recipient cells, did not possess general toxic effect and were not 

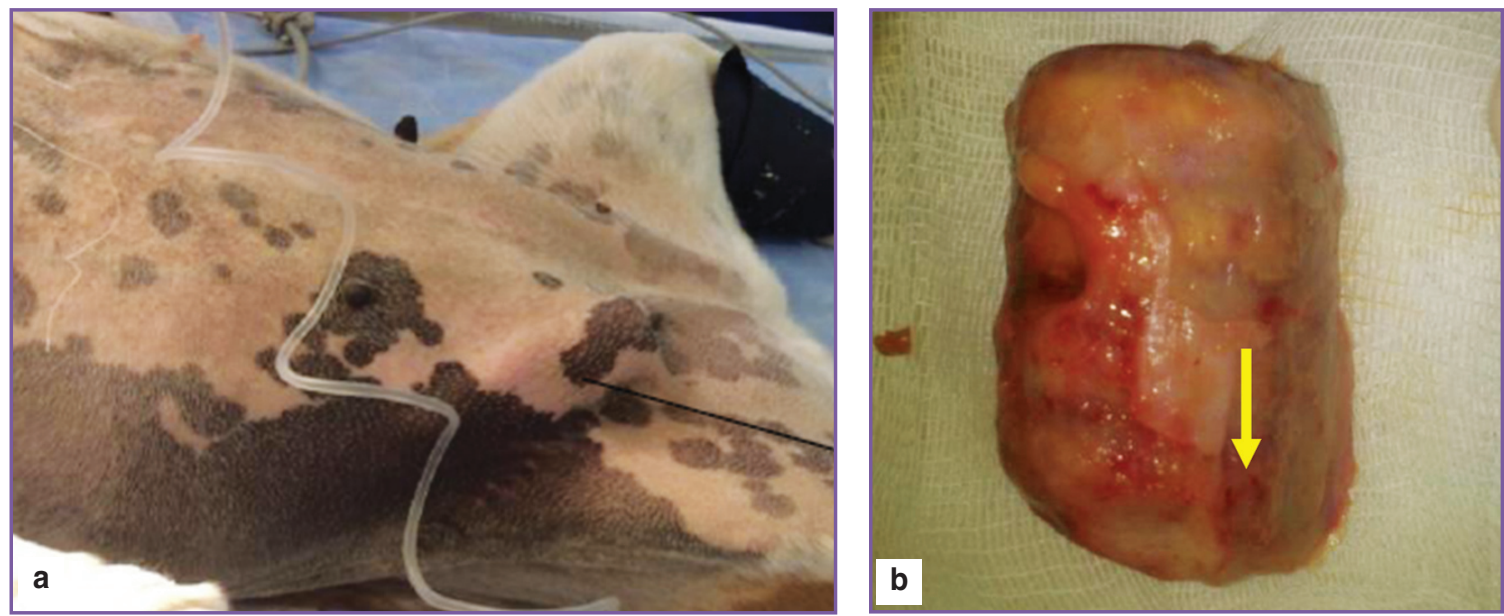

Figure 6. Synthetic tracheal matrix colonized by mesenchymal multipotent stromal cells 35 days after heterotopic transplantation: (a) inguinal area of the recipient dog after transplantation of the matrix specimen; (b) general view of the synthetic tracheal matrix after extraction from the recipient body tissues. Connective tissue capsule, intimately bound to the tracheal matrix, is indicated by the arrow

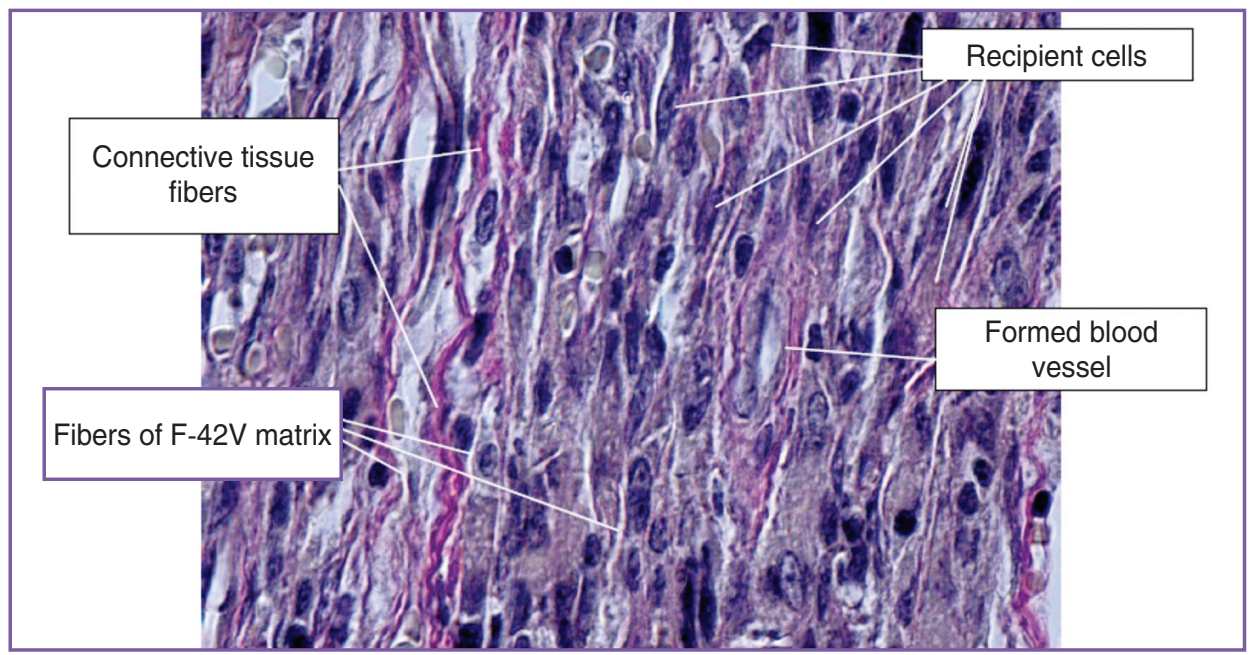

Figure 7. Active colonization of the fibers of the tracheal nonwoven matrix layer by the recipient cells

subject to biodegradation when exposed to the internal environment of the organism. These data allowed us to make a conclusion on the optimal compatibility of the developed matrices and their perspective application as bioimplants for the replacement of tracheal defects.

Study Funding. The work was financially supported by the Ministry of Education and Sciences of the Russian Federation (agreement on subsidy No.14.604.21.0023 of 17.06.2014, unique identifier of applied researches RFMEFI60414X0023).

Conflicts of Interest. The authors declare no conflicts of interest.

\section{References}

1. Kiselevsky M.V., Sitdikova S.M., Tenchurin T.Kh., Khomchenko A.U. Contemporary approaches and perspectives to creation of tracheal bioimplants. Rossiyskiy bioterapevticheskiy zhurnal 2014; 13(3): 127-131.

2. Kiselevsky M.K., Sitdikova S.M., Anisimova N.Yu., Polotsky B.E., Davidov M.I. Prospective synthetic matrixes for the reconstruction of defects of the trachea in patients with cancer. Voprosy onkologii 2015; 61(3): 323-328.

3. Del Gaudio C., Baiguera S., Ajalloueian F., Bianco A., Macchiarini $P$. Are synthetic scaffolds suitable for the development of clinical tissue-engineered tubular organs? J Biomed Mater Res A 2014; 102(7): 2427-2447, http://dx.doi. org/10.1002/jbm.a.34883.

4. Kiselevskiy M.V., Anisimova N.Yu., Lebedinskaya O.V., Polotskiy B.Ye., Davydov M.I. Heterotopic transplantation of non-immunogenic trachea populated with recipient bone marrow stromal cells. Morfologiia 2012; 141(1): 66-70.

5. Kopylov A.N., Anisimova N.Yu., Tenchurin T.Kh., Grigoriev T.E., Khomenko A.U., Kiselevsky M.V. Advanced materials to create a matrix of the trachea implantat. Rossiyskiy bioterapevticheskiy zhurnal 2014; 13(2): 67-71. 
6. da Silva T.H., Pazetti R., Aoki F.G., Cardoso P.F., Valenga M.H., Deffune E., Evaristo T., Pêgo-Fernandes P.M., Moriya H.T. Assessment of the mechanics of a tissue-engineered rat trachea in an image-processing environment. Clinics 2014; 69(7): 500-503, http://dx.doi.org/10.6061/clinics/2014(07)11.

7. Macchiarini $P$., Jungebluth $P$., Go T., Asnaghi M.A. Rees L.E., Cogan T.A., Dodson A., Martorell J., Bellini S., Parnigotto P.P., Dickinson S.C., Hollander A.P., Mantero S., Conconi M.T., Birchall M.A. Clinical transplantation of a tissueengineered airway. Lancet 2008; 372(9655): 2023-2030, http:// dx.doi.org/10.1016/S0140-6736(08)61598-6.

8. Crowley C., Birchall M., Seifalian A.M. Trachea transplantation: from laboratory to patient. J Tissue Eng Regen Med 2015; 9(4): 357-367, http://dx.doi.org/10.1002/ term.1847.

9. Omori K., Tada Y., Suzuki T., Nomoto Y., Matsuzuka T., Kobayashi K., Nakamura T., Kanemaru S., Yamashita M., Asato R. Clinical application of in situ tissue engineering using a scaffolding technique for reconstruction of the larynx and trachea. Ann Otol Rhinol Laryngol 2008; 117(9): 673-678, http://dx.doi.org/10.1177/000348940811700908.

10. Ajalloueian F., Lim M.L., Lemon G., Haag J.C., Gustafsson Y., Sjöqvist S., Beltrán-Rodríguez A., Del Gaudio C., Baiguera S., Bianco A., Jungebluth P., Macchiarini P. Biomechanical and biocompatibility characteristics of electrospun polymeric tracheal scaffolds. Biomaterials 2014; 35(20): 53075315, http://dx.doi.org/10.1016/j.biomaterials.2014.03.015.

11. Kolokolov R.G., Gerasina E.V., Anan'ev O.L. Polnyy spravochnik [Assays. Full directory]. Moscow: Eksmo; 2007; $786 \mathrm{p}$.

12. Bastl C.P., Hendler E.D., Finkelstein F.O. Leukocyte responses to acute renal transplant rejection. Clin Nephrol 1975; 4(6): 228-233.

13. de Fijter J.W. The impact of age on rejection in kidney transplantation. Drugs Aging 2005; 22(5): 433-449, http://dx.doi. org/10.2165/00002512-200522050-00007. 